\title{
Importance of Posterior Tibial Slope, Medial Tibial Plateau Slope and Lateral Tibial Plateau Slope in Anterior Cruciate Ligament Injury
}

\section{Bernat Mas Matas ${ }^{1}$, Irene Carrión Barberà2 ${ }^{2}$, Salvatore Marsico ${ }^{3}$, Anna Agustí Claramunt ${ }^{3}$, Raúl Torres-Claramunt ${ }^{4}$, Albert Solano López ${ }^{3}$}

${ }^{1}$ Universitat Pompeu Fabra, Universitat Autònoma de Barcelona, Barcelona, Spain

${ }^{2}$ Department of Rheumatology, Hospital del Mar, Passeig Marítim de la Barceloneta, Barcelona, Spain

${ }^{3}$ Department of Radiology, Hospital del Mar, Passeig Marítim de la Barceloneta, Barcelona, Spain

${ }^{4}$ Department of Orthopaedic Surgery and Traumatology, Hospital del Mar, Passeig Marítim de la Barceloneta,

Barcelona, Spain

Email: ${ }^{\star 40161 @ p a r c d e s a l u t m a r . c a t ~}$

How to cite this paper: Matas, B.M., Barberà, I.C., Marsico, S., Claramunt, A.A., TorresClaramunt, R. and López, A.S. (2021) Importance of Posterior Tibial Slope, Medial Tibial Plateau Slope and Lateral Tibial Plateau Slope in Anterior Cruciate Ligament Injury. Open Journal of Orthopedics, 11, 233-248.

https://doi.org/10.4236/ojo.2021.119022

Received: July 12, 2021

Accepted: September 3, 2021

Published: September 6, 2021

Copyright $\odot 2021$ by author(s) and Scientific Research Publishing Inc. This work is licensed under the Creative Commons Attribution International License (CC BY 4.0).

http://creativecommons.org/licenses/by/4.0/

\begin{abstract}
Increased posterior tibial slope (PTS), medial tibial plateau slope (MTPS) and lateral tibial plateau slope (LTPS) have been proposed as potential risk factors for anterior cruciate ligament (ACL) tear. However, prior literature is inconclusive. The primary goal of the study was to investigate the relationship of MTPS, LTPS and PTS with ACL tears. The secondary goal was to determine whether injury mechanisms, age, gender, tobacco, obesity, extreme PTS $\left(>12^{\circ}\right)$ or previous injuries are risk factors in ACL tears. Three groups were identified: a study group of patients with ACL injury; a second group diagnosed with meniscal injury; and a third group of non-injured patients. Demographic data was collected. MTPS and LTPS were measured in MRI and PTS in lateral radiographs by two blinded observers. Data was analyzed using SPSS. 416 patients were included in the study. MTPS ranged from $0^{\circ}$ to $14^{\circ}$ (average value $5.66 \pm 2.98)$, LTSA $0^{\circ}$ to $16^{\circ}(5.69 \pm 3.5)$ and PTS $0^{\circ}$ to $17^{\circ}$ (6.75 \pm 3.18$)$. Neither MTPS, LTPS nor PTS revealed increased risk of ACL tears related with higher slopes, although high MTPS showed a decreased risk. However, when comparing traumatic groups, extreme PTS was associated with LCA tear, but no differences were found when comparing non-traumatic groups. This could mean that the importance of extreme angles in the LCA rupture may be restricted to traumatic mechanisms. Previous ACL tear was identified as a risk factor to ACL tear; increased age and a traumatic mechanism were associated with a lower risk.
\end{abstract}




\section{Keywords}

ACL Tear, ACL Tear Risk Factors, Tibial Slope, Magnetic Resonance Imaging

\section{Introduction}

\subsection{Anatomy}

One of the first anatomical descriptions of the anterior cruciate ligament (ACL) can be found written on an Egypt papyrus scroll that dates to 3000BC. Hippocrates (460-370BC) described a subluxation of the human knee caused by injury of the ACL; however, the name of the ligament was applied by Claudius Galen of Pergamon as "ligamenta genu cruciate" (129-199BC) [1].

The ACL is a band of dense connective tissue that connects the femur and the tibia. It is enveloped in the synovial membrane of the human knee joint, which locates the ligament intra-articular but extrasynovial. The ligament originates at the medial side of the lateral femoral condyle and runs an oblique course through the intercondylar fossa distal, anterior and medial to the insertion at the medial tibial eminence. Due to its orientation within the knee joint, the ACL has been shown to be primary restraint to anterior tibial translation and secondary restraint to internal rotation of the weight bearing and non-weight bearing knee [2].

The width of the ACL ranges from $7 \mathrm{~mm}$ to $12 \mathrm{~mm}$. The ligament fans out toward its insertion at the tibia and the narrowest diameter can be found in the midsubstance area of the ACL [3]. The length of the ACL fibers ranges from 22 $\mathrm{mm}$ to $41 \mathrm{~mm}$ with a mean of $32 \mathrm{~mm}$. However, these measurements are more for the anteromedial bundle. Little is known about the intraarticular length of the posterolateral bundle [4].

The ACL is made of several collagen bundles that give rise to the multifibrillar nature of the ligament, whose femoral and tibial insertions, as well as spatial orientation in the knee, are directly related to its function as the fulcrum of joint movement [4] [5] [6].

\subsection{Biomechanics}

The anterior cruciate ligament plays a crucial role in stabilizing the knee joint; in the sagittal plane, it is the main static stabilizer and prevents anterior translation of the tibia on the femur. The role that the ACL plays in rotational stability is less well known, although it is known that during the flexion extension movement, the ligament presents a degree of tension that makes severe degrees of hyperextension of the joint impossible and plays a secondary stabilizing role in varo-valgus movements [7].

The knee joint serves as an important structure that supports the full body weight during daily locomotion; hence, it is most susceptible to acute impact injuries. Based on previous reports, $40 \%$ of sports-related injuries were noted to be 
located at the knee joint and be highly prevalent in intensive sports, with a wide spectrum of mechanisms in which injury will occur [7] [8] [9] [10].

Different authors such as Frank et al. [11] and Mokhtarzadeh et al. [12] expose the importance of other factors that may favor ACL injury. The first affirms that the muscles of the trunk and the pelvis play a fundamental factor; the second speaks of the relationship of agonistic or antagonistic muscles in relation to stability in the sagittal plane and mentions the importance of the soleus and gastrocnemius.

Currently, the research is based on the creation of finite elements that can reproduce situations of maximum stress to assess individual contribution of each possible risk factor.

Although ACL injuries can occur during a wide variety of activities, a few mechanisms account for most ACL tears. The most common mechanisms of ACL injury and the structures affected are [10]:

Pivot shift injury: characterized by valgus loading, flexion and external rotation of the tibia, or internal rotation of the femur. This mechanism characteristically causes contusions of the lateral compartment and is associated with meniscal and posterolateral capsular injury.

Hyperextension injury: determined by the force directed to the anterior tibia with a planted foot. A Kissing contusion is seen in the anterior femoral condyle and the anterior tibial plateau. Associated soft tissue injuries include meniscal and PCL lesions.

Clip injury: secondary contact injury to pure valgus stress on a partially flexed knee. Many patients have medial collateral ligament and meniscal injuries.

Dashboard injury: caused by force on the anterior proximal tibia with the knee in flexion and is associated with anterior tibial and posterior patellar edema as well as rupture of the PCL and the posterior joint capsule.

\subsection{Anatomical Factors that May Increase the Risk of ACL Injury}

There are many anatomical factors involved in the risk of ACL injuries. The most important are: the intercondylar notch index, the intercondylar notch height index, $\alpha$ angle (notch angle), $\beta$ angle (formed by the Blumensaat line and the long axis of the femur), medial tibial plateau slope and lateral tibial plateau slope. Although there is no absolute agreement, there are three factors that seem to be most involved: [12]

A narrower intercondylar notch $<0.252$. The notch width index (NWI) is used for the ratio of epicondylar width to notch width. Since patients with bilateral ACL tear showed a smaller NWI, the risk of sustaining an ACL tear could be higher with a smaller NWI [1] (Figure 1).

A $\beta$ angle $>38.5^{\circ}$ can cause the ACL to be more horizontal, thereby causing greater ACL impingement against the anterior intercondylar notch, leading to more ACL injury (Figure 1). Alentorn-Geli [13] pointed out that the B angle might be the most important isolated factor in ACL injury, but they proposed 


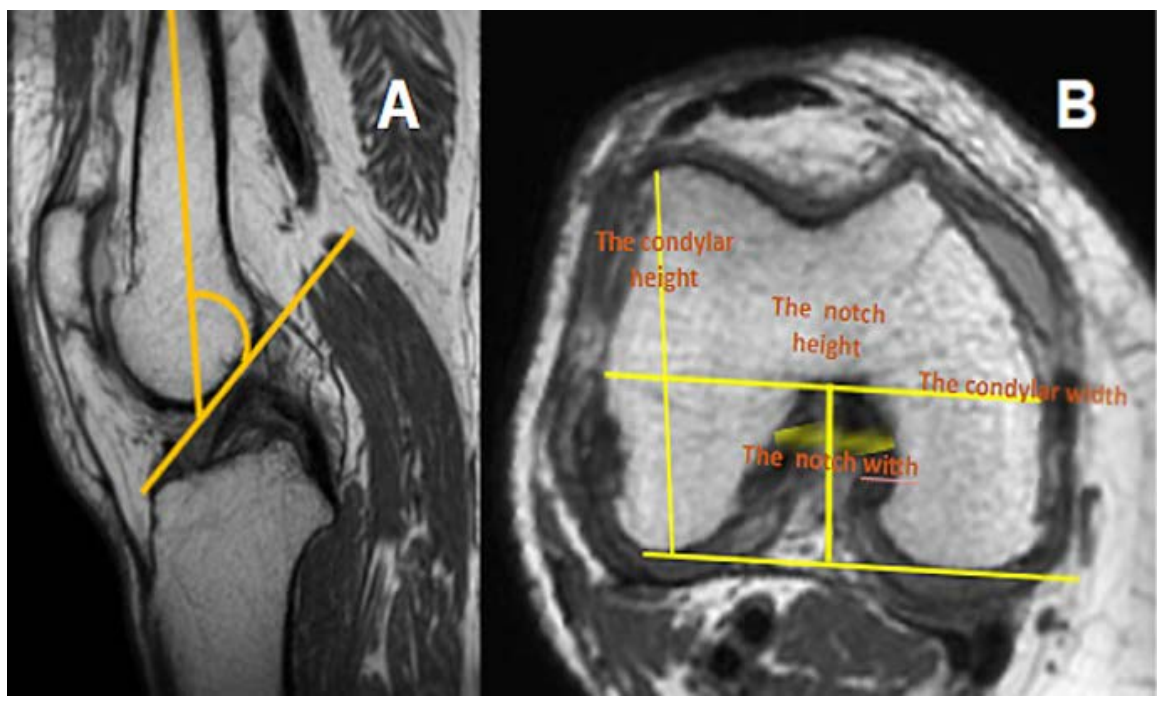

Figure 1. $\beta$ angle ((A) T1 Turbo Spin Echo (TSE) image in sagittal plane) and intercondylar notch width ((B) T1 TSE image in axial plane). An increased and a reduced intercondylar notch width are identified risk factors to LCA tear.

that the risk for ACL injury is multifactorial.

An increased lateral tibial slope. Different studies have shown a connection between ACL injury and tibial slope, but the results are controversial. Biomechanically, an increase in the tibial slope can create greater translational force which could result in an increase in the anterior translational movement of the tibia. It is recognized that a critical role of the ACL is to prevent anterior tibial translation. Thus, an increased tibial slope may lead to increased tension of the ACL and even ACL tear [14] [15].

The great variability of slope values in different studies is a difficulty to establish normal values of PTS, MTPS and LTPS, that could be different during lifetime and change different depending on the sex. Furthermore, in most articles, there is a bias, as most patients are Caucasian, which prevents the small number of patients enrolled in the studies from determining whether there is variation in PTS depending on race [14] [16] [17] [18].

\subsection{Incidence, Distribution by Sex and by Sports Activity}

The prevalence is 68.6 per 100,000 person-year [19]. Incidence is significantly higher in male patients than in females $(81.7$ vs 55.3 per $100,000, \mathrm{P}<0.001)$. Age-specific patterns differed in male and female patients, with a peak in incidence $(241.0$ per 100,000$)$ between 19 and 25 years in males and a peak in incidence $(227.6$ per 100,000) between 14 and 18 years in females [20].

Sport activity is related to LCA ruptures. Professional athletes in basketball, soccer, and the other football codes report an annual incidence of $0.15 \%-3.7 \%$ in studies with at least a moderate sample size. Annual ACL incidence in amateur sporting groups was generally higher than the entire population but lower than among professional athletes. 


\section{Materials and Methods}

\section{A Multicenter Retrospective Study Was Performed.}

Sixty-eight patients with a history of anterior cruciate ligament (ACL) tear and eighty-six patients with a history of meniscal injuries who underwent surgery in Parc de Salut Mar from January 2015 to December 2019 were identified from the clinical database. Another group of healthy non-injured patients was also reviewed as a control group in both MRI and lateral radiography. It was composed of 282 patients from the cohort of non-injured patients who underwent MRI or X-Ray in Hospital del Mar. Knee MRI and lateral knee radiographs from the three groups were analyzed using Centricity Universal Viewer software (General Electric (GE) Healthcare's, Wauwatosa, WI) on the Hospital database and Raim Viewer software on Primary Attention tests. Angular values reported by the software were single digit.

X-ray and MRI imaging exclusion criteria were [17] [21] [22] [23]:

- MRI or X-ray image with artifacts (related to prosthesis, metal device, movement in MRI or alteration of the digital detail in X-ray) that do not allow adequate retrospective study.

- Magnetic resonance or X-ray image that does not meet general quality criteria (wrong FoV that does not include the structures to be examined in the knee, including sagittal plane not perpendicular to the principal axis, altered saturation of the MR image due to proximity of the coil).

- Measurements made on lateral knee radiographs must meet the quality inclusion criteria: the femoral condyles should be perfectly superimposed or the posterior condyle borders within maximum $5 \mathrm{~mm}$ of distance.

No further requirements were placed on age, skeletal maturity, or sex. Medical records were analyzed to determinate injury mechanism, age, sex, obesity, laterality, smoking status and presence of previous injuries to prepare a multivariate analysis.

MTPS and LTPS were measured by the Hashemi et al. method [22] (Figure 2). Both were defined as the angle between the $90^{\circ}$ line with the proximal tibial anatomic axis and a tangential line drawn along either plateau.

The anatomic axis of the tibia was calculated measuring two perpendicular lines which included all tibial cortex, established 3 and $4 \mathrm{~cm}$ from the tibial plateau. The drawn line passing through the midpoint of both perpendicular lines is defined as the anatomic axis.

In lateral radiographs, proximal anatomic axis was calculated measuring two perpendicular lines which included all tibial cortex, established $5 \mathrm{~cm}$ below tibial tuberosity and $15 \mathrm{~cm}$ distal from the tibial plateau. The drawn line passing through the midpoint of both perpendicular lines is defined as the anatomic axis. PTS was defined as the tangential between this axis and the line drawn along the plateau [17] (Figure 3).

There are different radiological methods of measurement for PTS. Regardless of the imaging technique used, 4 are the basic methods of radiological measurement 


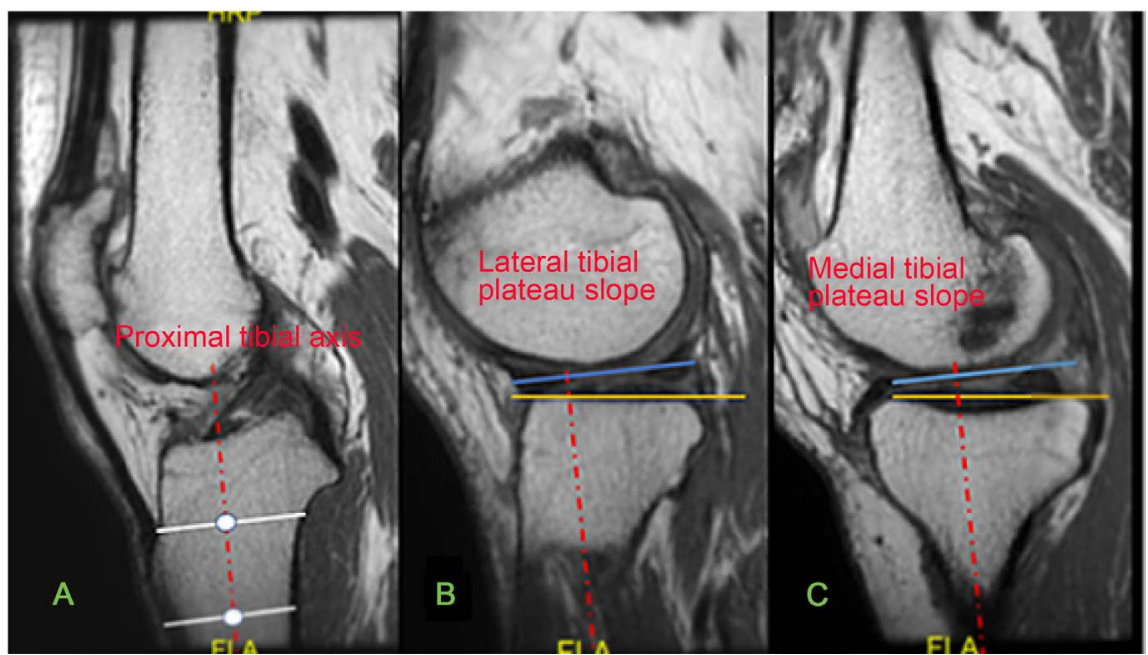

Figure 2. Measures of MTPS and LTPS MRI - T1 TSE sequences in the sagittal plane. MTPS and LTPS were defined as the angle between the $90^{\circ}$ line (blue lines in (B) and (C)) with the proximal tibial anatomic axis (spotted red line) and a tangential line drawn along either plateau (yellow line in (B) and (C)). The anatomic axis of the tibia was calculated measuring two perpendicular lines which included all tibial cortex (white horizontal lines in (A)). The drawn line passing through the midpoint (white dots in (A)) of both perpendicular lines is defined as the anatomic axis (spotted red line).

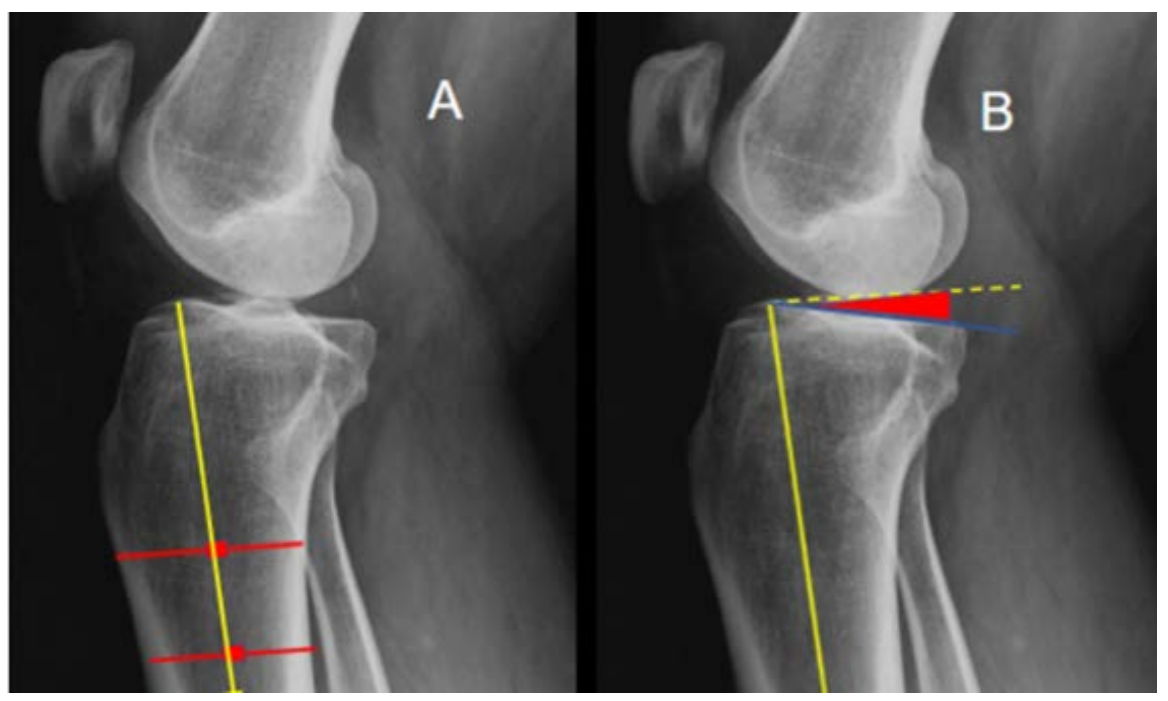

Figure 3. Measures in lateral projection on conventional radiography: proximal anatomic axis (PTAA). Proximal anatomic axis (PTAA) was calculated measuring two perpendicular lines which included all tibial cortex, established 5 and $15 \mathrm{~cm}$ distal from the tibial plateau (red lines in (A)). The drawn line passing through the midpoint of both perpendicular lines (red squares) is defined as the anatomic axis (yellow vertical line in (A) and (B)). PTS (red angle) was defined as the tangential (spotted yellow line) between this axis and the line drawn along the plateau (blue line).

of tibial slope: anterior tibial cortex (ATC), proximal tibial anatomic axis (PTAA), posterior tibial cortex (PTC), and the Hudek method. All these methods use the tangent to the medial tibial plateau as the proximal reference line, but this medial line is calculated differently. In ATC, this line matches exactly 
with the anterior tibial cortex, starting on the most distal point available; PTAA is described previously as this is the method used in the current study; PTC uses the line matching the posterior tibial cortex and Hudek defined this line as the one crossing both midpoints of circles previously drawn. The cranial circle touches the anterior, posterior, and cranial tibial cortex and the caudal one touches both anterior and posterior cortex. The angle between the tangent to the medial plateau and the anatomic line of the plateau is defined as the angle in all methods (Figure 4 and Figure 5), [17] [21] [23] [24] [25].

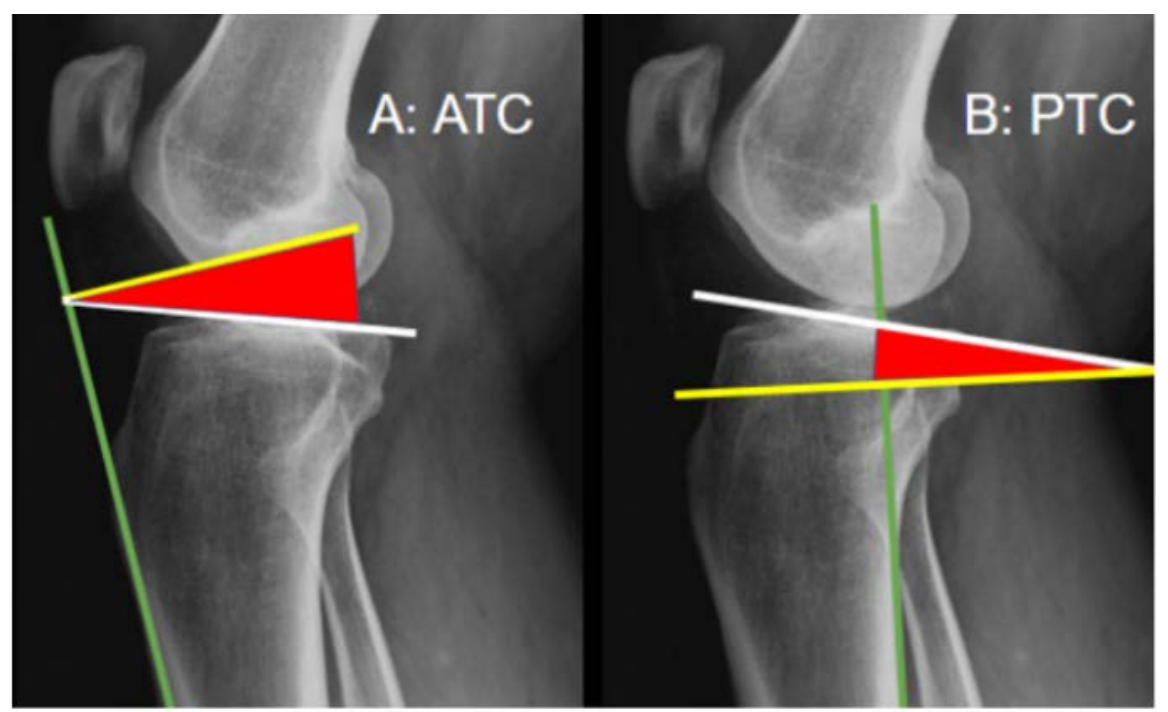

Figure 4. Anterior tibial cortex (ATC) and posterior tibial cortex (PTC) measurement methods for PTS in lateral radiography. ATC (A) and PTC (B) measure methods are shown in the figure. First, the proximal reference line (green line in (A) and (B)) is defined as the line passing through the anterior tibial cortex in ATC and the posterior tibial cortex in PTC. The tangent to that line is drawn (yellow line) and the angle formed by this tangent and the anatomic plateau line (white line) is defined as the angle (red angle).

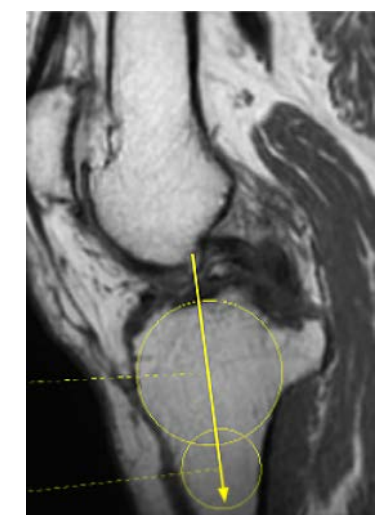

Figure 5. Hudek et al., 2009 measurement method - T1 TSE image in sagittal plane. This method uses the tangent to the medial tibial plateau as the proximal reference line, defined as the one crossing both midpoints of circles previously drawn. The cranial circle touches the anterior, posterior and cranial tibial cortex and the caudal one touches both anterior and posterior cortex. The angle between the tangent to the tibial axis and the anatomic line of the plateau is defined as the angle in both medial and lateral plateaus. 
For the descriptive statistics, quantitative variables were described through mean and standard deviation and qualitative variables through number and percentage. Bivariate analysis was performed to identify factors associated with ACL injury. Qualitative variables were compared by Chi-square or Fisher exact test. Fisher's exact test was used when $>20 \%$ of the cells in $2 \times 2$ contingency table had a frequency $<5$. Group comparison of quantitative variables was performed by Student's t-test and correlation of quantitative variables through Pearson's correlation coefficient. A multivariate analysis was performed with variables suspicious to influence in ACL injury through a logistic regression analysis.

Statistical analysis was performed using IBM ${ }^{\otimes}$ SPSS 25.0. Results were considered as statistically significant at p-value $<0.05$.

Twenty-five perioperative MRI were initially reviewed twice, separated by five weeks, by two different blinded observers. Intra and interobserver correlations coefficients (ICC) and their 95\% confidence intervals were calculated based on a mean-rating $(\mathrm{k}=2)$, consistency, 2-way random-effects model.

The protocol for our research was consistent with the provisions of the World Medical Association Declaration of Helsinki and the legal rules on the confidentiality of data were followed. This study (code 2021/9752) has a provisional approval by the Clinical Research Ethical Committee of Parc de Salut Mar, pending its final approval.

\section{Results}

Patients who did not match inclusion criteria or match any exclusion criteria (including absence of X-Ray and MRI) (fifteen meniscal injuries, five ACL) on the Hospital or Primary Attention database were excluded from the two study groups. In sum, twenty patients were excluded, leaving sixty-three ACL tears and seventy-one meniscal injury patients suitable for the study. 282 patients were included in the non-injured control group.

The inter-observer agreement was excellent in both MTPS [0.971 (IC 95\% 0.927 - 0.989)] and LTPS [0.970 (IC 95\% 0.925 - 0.988)]. The intra-observer agreement for rater one was good in MTPS 0.885 (IC 95\% 0.711 - 0.955) and excellent in LTPS, [ICC 0.971 (IC 95\% 0.929 - 0.989)]; for the rater two was excellent in both sides, MTPS 0.980 (IC 95\% 0.948 - 0.992) and LTPS ICC 0.987 (IC $95 \% 0.968-0.995)$. The data selected for the statistical analysis were the average measures by rater 1 .

Demographic characteristics of patients by group are shown in Table 1 . Maximum, minimum and average MTPS, LTPS and PTS measures per group are shown in Table 2.

Significant differences between groups were identified in age. ACL tear patients are younger than in the other groups (Table 1). Other significant differences were found between groups in sex, obesity, smoking, previous injuries, and injury mechanism. Of these, only previous injuries, specifically previous 
Table 1. Demographic characteristics of study groups.

\begin{tabular}{|c|c|c|c|c|}
\hline & $\begin{array}{c}\text { ACL Injury } \\
(\%)\end{array}$ & $\begin{array}{c}\text { Meniscal injury } \\
(\%)\end{array}$ & $\begin{array}{l}\text { Healthy control } \\
\text { (\%) }\end{array}$ & $\begin{array}{c}\text { All groups } \\
\text { (\%) }\end{array}$ \\
\hline $\begin{array}{l}\text { Number of } \\
\text { patients }\end{array}$ & $63(15.1)$ & $71(17.1)$ & $282(77.8)$ & $416(100.0)$ \\
\hline \multicolumn{5}{|c|}{ Gender } \\
\hline Male & $41(65.1)$ & $52(73.2)$ & $156(55.3)$ & $249(59.9)$ \\
\hline Female & $22(34.9)$ & $19(26.8)$ & $126(44.7)$ & $167(40.1)$ \\
\hline \multicolumn{5}{|c|}{ Smoking Status } \\
\hline No smoker & $51(81.0)$ & $43(60.6)$ & $225(79.8)$ & $319(76.7)$ \\
\hline Smoker & $12(19.0)$ & $28(39.4)$ & $57(20.2)$ & $97(23.3)$ \\
\hline \multicolumn{5}{|c|}{ Obesity } \\
\hline No obese & $57(90.5)$ & $57(80.3)$ & $256(90.8)$ & $370(88.9)$ \\
\hline Obese & $6(9.5)$ & $14(19.7)$ & $26(9.2)$ & $46(11.1)$ \\
\hline \multicolumn{5}{|c|}{ Injury mechanism } \\
\hline Non-traumatic & $48(76.2)$ & $61(85.9)$ & $151(53.5)$ & $260(62.5)$ \\
\hline Traumatic & $15(23.8)$ & $10(14.1)$ & $131(46.5)$ & $156(37.5)$ \\
\hline \multicolumn{5}{|c|}{ Previous injuries } \\
\hline None & $34(54.0)$ & $53(74.6)$ & $275(97.5)$ & $362(87.0)$ \\
\hline ACL tear & $29(46.0)$ & $4(5.6)$ & $0(0.0)$ & $33(1.9)$ \\
\hline Meniscal injury & $0(0.0)$ & $14(19.7)$ & $7(2.5)$ & $21(5.0)$ \\
\hline Age $^{*}$ & $31.95 \mathrm{y}$ & $45.23 \mathrm{y}$ & 40.52 y & $40.03 \mathrm{y}$ \\
\hline
\end{tabular}

*Age in years: LCA tear group: Minimum 14, maximum 64 and average 31.95; Meniscal injury group: Minimum 7, maximum 70 and average 45.23; Healthy control: Minimum 10, maximum 86 and average 40.52; All groups: Minimum 7, maximum 86 and average 40.03. ACL: Anterior cruciate ligament; y: Years.

Table 2. Medial tibial plateau slope (MTPS), lateral tibial plateau slope (LTPS) and posterior tibial slope (PTS) measures by group.

\begin{tabular}{cccccccccc}
\hline \multirow{2}{*}{ Group } & \multicolumn{4}{c}{ MTPS } & \multicolumn{3}{c}{ LTPS } & \multicolumn{3}{c}{ PTS } \\
\cline { 2 - 10 } & Min & Max & Average & Min & Max & Average & Min & Max & Average \\
\hline All patients & $0^{\circ}$ & $14^{\circ}$ & $5.66 \pm 2.98$ & $0^{\circ}$ & $16^{\circ}$ & $5.69 \pm 3.5$ & $0^{\circ}$ & $17^{\circ}$ & $6.75 \pm 3.18$ \\
ACL tear & $0^{\circ}$ & $12^{\circ}$ & $4.98 \pm 2.62$ & $1^{\circ}$ & $14^{\circ}$ & $5.87 \pm 2.97$ & $0^{\circ}$ & $16^{\circ}$ & $6.62 \pm 3.3$ \\
$\begin{array}{c}\text { Meniscal } \\
\text { injury }\end{array}$ & $0^{\circ}$ & $13^{\circ}$ & $6.1 \pm 3.13$ & $0^{\circ}$ & $16^{\circ}$ & $6.92 \pm 3.95$ & $0^{\circ}$ & $15^{\circ}$ & $7,03 \pm 2.93$ \\
Control & $0^{\circ}$ & $14^{\circ}$ & $5.72 \pm 3.01$ & $0^{\circ}$ & $15^{\circ}$ & $5.18 \pm 3.39$ & $0^{\circ}$ & $17^{\circ}$ & $6.7 \pm 3.22$ \\
\hline
\end{tabular}

ACL: anterior cruciate ligament; Min: Minimum, Max: Maximum.

ACL tear $(\mathrm{p}=0.001)$, age (younger patients) $(\mathrm{p}=0.001)$ and injury mechanism $(\mathrm{p}=0.015)$ were associated with ACL tear. Regarding the injury mechanism, the traumatic mechanism was found to be less related with ACL tear than non-traumatic injury (OR: 0.47 IC $95 \% 0.25-0.87$ ).

No differences were found in the presence of extreme slopes regarding ACL group except in PTS when comparing traumatic control group and traumatic ACL tear group. Differences in extreme MTPS and LTPS were found when 
comparing the non-traumatic control and non-trauma-tic meniscal injury group, but this was not associated with ACL rupture. By radiography no extreme PTS were identified in either traumatic control or traumatic meniscal injury groups $\left(\mathrm{N}\right.$ PTS $\left.>12^{\circ}=0\right)($ Table 3$)$.

When analyzing the association between angles and different demographic and clinical characteristics of the patients, significant higher general angles by the three methods and significant differences in extreme MTPS and LTPS values were found in non-smokers $(\mathrm{p}<0.05)$. There were no smoking patients with extreme MTPS and only one extreme LTPS.

Regarding presence of extreme angles, significant differences were identified in PTS in sex and injury mechanism, with a higher presence in women and in non-traumatic injury group (Table 3 ).

A multivariate analysis was performed. Neither of the three measure methods revealed an increased risk for ACL tear related to higher or extreme angles, although an association between MTPS and a decreased risk of ACL rupture was identified OR: 0.869 95\% IC 0.778 - 0.972 ( $\mathrm{p}=0.014$ ). In all measure methods, previous injury (previous ACL tear) was related to a higher risk to ACL rupture; increased age and traumatic mechanism lowered tear risk.

Pearson's correlation coefficient between medial and lateral MRI angle was moderate $(0.577)$, but better than medial MRI and PTS by radiography, which

Table 3. Presence of extreme angles in the different groups.

\begin{tabular}{|c|c|c|c|c|c|c|c|}
\hline & $\begin{array}{l}\text { \# of } \\
\text { pt. }\end{array}$ & $\begin{array}{c}\text { \# of } \\
\text { extr. } \\
\text { MTPS }\end{array}$ & $\begin{array}{l}\% \text { of } \\
\text { extr. } \\
\text { MPTS }\end{array}$ & $\begin{array}{l}\text { \# of } \\
\text { extr. } \\
\text { LTPS }\end{array}$ & $\begin{array}{l}\% \text { extr. } \\
\text { LTPS }\end{array}$ & $\begin{array}{l}\text { \# of } \\
\text { extr. } \\
\text { PTS }\end{array}$ & $\begin{array}{c}\% \\
\text { extr. } \\
\text { PTS }\end{array}$ \\
\hline All patients & 416 & 8 & 1.9 & 19 & 4.6 & 21 & 5.0 \\
\hline ACL tear group & 63 & 1 & 1.6 & 3 & 4.8 & 5 & 7.9 \\
\hline \multicolumn{8}{|c|}{ Injury mechanism } \\
\hline Non-traumatic & 48 & 1 & 2.1 & 1 & 2.1 & 3 & 6.3 \\
\hline Traumatic & 15 & 0 & 0 & 2 & 13.3 & 2 & 13.3 \\
\hline $\begin{array}{l}\text { Meniscal injury } \\
\text { group }\end{array}$ & 71 & 2 & 2.8 & 9 & 12.7 & 4 & 5.6 \\
\hline \multicolumn{8}{|c|}{ Injury mechanism } \\
\hline Non-traumatic & 61 & 2 & 3.3 & 9 & 14.8 & 4 & 6.6 \\
\hline Traumatic & 10 & 0 & 0 & 0 & 0 & 0 & 0 \\
\hline $\begin{array}{l}\text { Healthy control } \\
\text { group }\end{array}$ & 282 & 5 & 1.8 & 7 & 2.5 & 12 & 4.3 \\
\hline \multicolumn{8}{|c|}{ Injury mechanism } \\
\hline Non-traumatic & 151 & 0 & 0 & 3 & 2.0 & 12 & 7.9 \\
\hline Traumatic & 131 & 5 & 3.8 & 4 & 3.1 & 0 & 0 \\
\hline
\end{tabular}

\# of pt.: number of patients; \# of extr. MTPS: number of extreme MTPS; \% of extr. MTPS: percentage of extreme MTPS; \# of extr. LTPS: number of extreme LTPS; \% of extr. LTPS: percentage of extreme LTPS; \# of extr. PTS: number of extreme PTS; \% of extr. PTS: percentage of extreme PTS. ACL: anterior cruciate ligament. 
was weak (0.387). Lateral MRI angle and radiography have a strong correlation coefficient (0.617) (Figure 6).

\section{Discussion}

The results of our study revealed that PTS, MTPS or LTPS were not associated with an increased ACL tear risk in multivariate analysis, but significance was found in PTS measured by radiography associating an extreme angle with rupture in traumatic ACL rupture patients when compared to traumatic control group. Increased MTPS is identified as a protector factor against ACL tears OR: $0.86995 \%$ IC $0.778-0.972$. This is not consistent with previous literature, which suggests that this slope could also be a risk factor for ACL tear just as the LTPS, even though not all literature agrees with this information and there is controversy even on meta-analysis, suggesting that there is not enough information to accept MTPS as a risk factor for ACL tear [14] [15]. This study shows not enough significant difference to support MTPS, LTPS or PTS individually as a risk factor for ACL tear.

Other risk factors identified in this study to ACL tear are the younger age, non-traumatic injury mechanism and previous ACL injuries.

The young age could be a risk factor as the biomechanics of the knee change while growing. Recent studies show that there is a variation in the value of PTS throughout life, first observing a decrease and then an increase, even though the point at which these changes occur is not the same in both sexes and could be different in both knees [8] [16]. The young age as a risk factor could also be related to injury mechanism, as ACL tears are highly prevalent in a broad range of intensive sports, including basketball, volleyball, soccer, and gymnastics, which

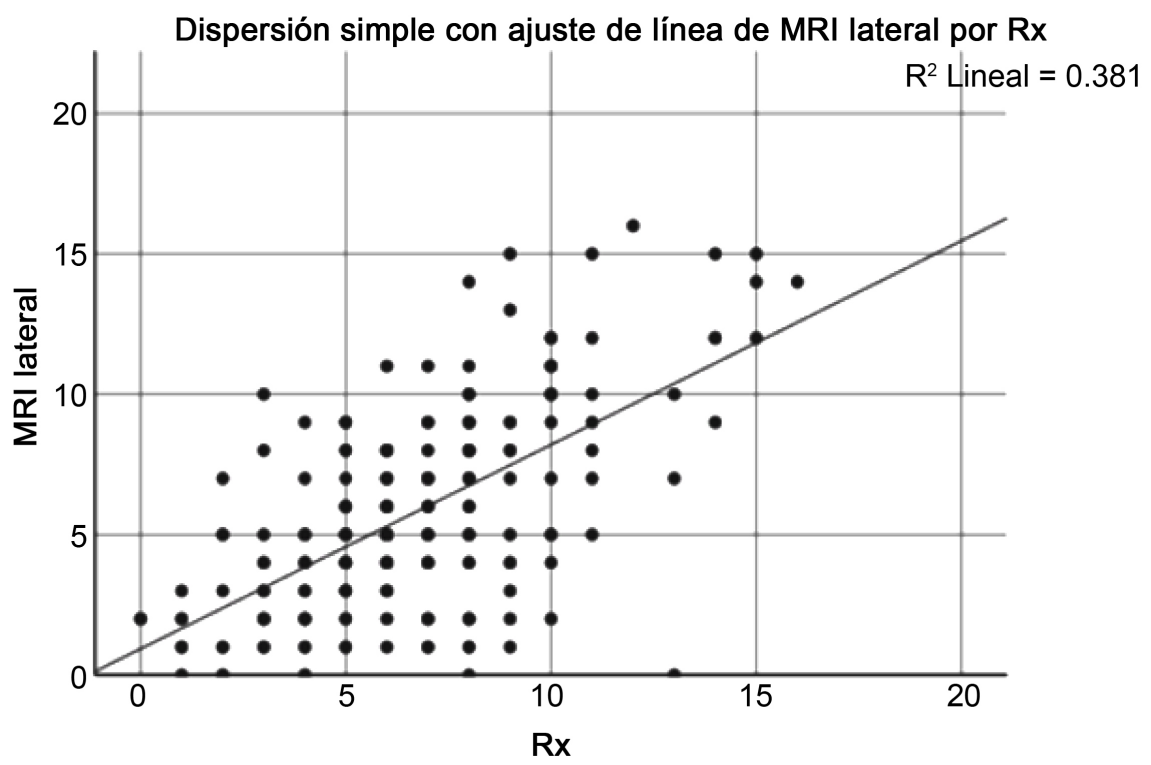

Filtrado por la variable Grup

Figure 6. Dispersion graphic of correlation between LTPS measured by MRI and PTS measured by lateral radiography. 
are much more practiced by younger people as they require intense athletic capacity. The risk of knee injury was found to be 10 times higher in competitive and recreational sports, typically practiced by younger patients, than in quiet life, widely distributed among all ages [10].

Non-traumatic injuries are identified in the study as a risk factor when compared to traumatic injuries, which is consistent with prior literature, where most ACL tears are related to maneuvers characterized by the presence of large ground impact, sudden deceleration, and changes in direction. These injury mechanisms can be classified based on at least the following key factors: ground impact, anterior tibial load, hyperextension moment, axial tibial torque, and valgus moment [7] [8].

Acute treatment of LCA injury consists of "RICE" therapy, which includes rest, ice, compression of the affected knee, and lifting of the affected lower limb.

Patients must be non-load bearing and can use crutches or a wheelchair if necessary. Pain relief can be achieved with over-the-counter medications such as NSAIDs but is typically at the discretion of the treating physician.

ACL injuries, depending on their severity, can be managed nonsurgically or operatively. The patient with an anterior cruciate ligament injury should be referred to the orthopedic doctor to discuss treatment options and a physical therapist (PT) for rehabilitation.

Non-surgical treatment is generally reserved for those with low functional demands, type and severity of the ACL injury, time of injury and subsequent evaluation. Continuous monitoring and treatment by an orthopedist and physical therapist are necessary and will only improve their functional status and post-injury stability. It should be noted that about half of patients who initially choose the non-surgical route will later choose to undergo surgical repair [25].

The results of the study show that previous ACL rupture is also an important risk factor to ACL tears. Other studies have linked an injury in the ACL with future injuries: graft ruptures (6.4\%) and contralateral ACL rupture (5.7\%). The highest risk of re-injury is four to six months after reconstruction. In another study, 70\% of ACL re-injury in elite footballers occurred in the same leg as previous injury [26] [27].

Significant higher general angles and differences in extreme MTPS and LTPS were found in non-smokers when compared to smokers. This possible protective factor of smoking towards the slopes is not mentioned in the literature at all, so further studies are needed to explore this possible association.

The differences found in extreme PTS regarding traumatic ACL tear group compared with traumatic control group but not in non-traumatic groups could be pointing that the importance of extreme PTS is restricted to traumatic injury mechanisms. Further studies should be carried to demonstrate this.

The range PTS values in the study ranged from $0^{\circ}$ to $17^{\circ}$ (average value $6.75 \pm$ 3.18) (Table 2). As seen in previous literature, the great variability of the values obtained is a difficulty to establish normal values, but the results obtained are included in the wide variability range (Table 4) [14] [15] [17] [26] [28] [29]. 
Table 4. Variability of Medial tibial plateau slope (MTPS), lateral tibial plateau slope (LTPS) and posterior tibial slope (PTS) in different studies.

\begin{tabular}{|c|c|c|c|c|c|c|}
\hline Authors & Journal & Group & MPTS av. & LPTS av. & $\begin{array}{c}\text { PTS } \\
\text { Min-Max }\end{array}$ & PTS av. \\
\hline \multirow{4}{*}{$\begin{array}{c}\text { Mas et al. } \\
\text { (Current Study) }\end{array}$} & \multirow{4}{*}{ - } & All patients & $5.66^{\circ} \pm 2.98^{\circ}$ & $5.69^{\circ} \pm 3.5^{\circ}$ & $0^{\circ}-17^{\circ}$ & $6.75^{\circ} \pm 3.18^{\circ}$ \\
\hline & & ACL tear & $4.98^{\circ} \pm 2.62^{\circ}$ & $5.87^{\circ} \pm 2.97^{\circ}$ & $0^{\circ}-16^{\circ}$ & $6.62^{\circ} \pm 3.3^{\circ}$ \\
\hline & & Meniscal injury & $6.1^{\circ} \pm 3.13^{\circ}$ & $6.92^{\circ} \pm 3.95^{\circ}$ & $0^{\circ}-15^{\circ}$ & $7.03^{\circ} \pm 2.93^{\circ}$ \\
\hline & & Healthy Control & $5.72^{\circ} \pm 3.01^{\circ}$ & $5.18^{\circ} \pm 3.39^{\circ}$ & $0^{\circ}-17^{\circ}$ & $6.7^{\circ} \pm 3.22^{\circ}$ \\
\hline Genin et al. & J Radiol & Healthy Control & - & - & $0^{\circ}-18^{\circ}$ & $7^{\circ}$ \\
\hline Jiang et al. & $\begin{array}{l}\text { J Formos } \\
\text { Med Assoc }\end{array}$ & Healthy and arthrosic & - & - & $0^{\circ}-20^{\circ}$ & $10^{\circ} \pm 4^{\circ}$ \\
\hline Dean et al. & J Arthro & Healthy & - & - & - & $11.8^{\circ} \pm 3.1^{\circ}$ \\
\hline Chiu et al. & J Arthro & Cadaveric & $14.8^{\circ}$ & $11.88^{\circ}$ & - & $11.58^{\circ}$ \\
\hline $\begin{array}{l}\text { Woderman et al. } \\
\text { (meta-analysis }\end{array}$ & Am J Sports & Healthy control & $\begin{array}{c}2.9^{\circ} \pm 2.80^{\circ} \text { to } \\
9.5^{\circ} \pm 3^{\circ}\end{array}$ & $\begin{array}{c}0.3^{\circ} \pm 3.6^{\circ} \text { to } \\
9^{\circ} \pm 4^{\circ}\end{array}$ & - & - \\
\hline $\begin{array}{l}\text { including } 14 \\
\text { articles) }\end{array}$ & Med & ACL tear & $\begin{array}{c}1.8^{\circ} \pm 3.5^{\circ} \text { to } \\
12.1^{\circ} \pm 3.3^{\circ}\end{array}$ & $\begin{array}{l}1.8^{\circ} \pm 3.2^{\circ} \text { to } \\
11.5^{\circ} \pm 3.54^{\circ}\end{array}$ & - & - \\
\hline Hudek et al. & $\begin{array}{l}\text { Clin Orthop } \\
\text { Relat Res }\end{array}$ & Healthy & $4.8^{\circ} \pm 2.4^{\circ}$ & $5^{\circ} \pm 3.6^{\circ}$ & - & $8.2^{\circ} \pm 2.8^{\circ}$ \\
\hline
\end{tabular}

Av: Average; Min-Max: minimum and maximum; ACL: anterior cruciate ligament; J Radiol: European Journal of Radiology; J Formos Med Assoc: Journal of the Formosan Medical Association; J Arthro: Journal of Arthroplasty; Am J Sports Med: The American Journal of Sports Medicine; Clin Orthop Relat Res: Clinical Orthopaedics and Related Research.

Regarding MTPS and LTPS, this study shows that MTPS values ranged from $0^{\circ}$ to $14^{\circ}$ (average value $5.66 \pm 2.98$ ) and LTSA $0^{\circ}$ to $16^{\circ}$ (average value $5.69 \pm$ 3.5). The values found are included and consistent with the extreme variability found in prior literature (Table 4). The methods used in this study are the same as in the others. However, results are still different, pointing to a need to unify the techniques and provide a gold standard which could guarantee a good interrater reliability [14] [23] [29].

Correlation between the PTS measured by lateral radiography and LTPS by MRI are strong, with higher PTS in radiographic measures (Figure 3). This is consistent with prior literature and what Lee et al. and Hudek et al. found measuring in the same patients using both systems [14] [21] [25] [30] [31].

Several limitations were identified. This study is based on real population. $\mathrm{Pa}$ tients cannot be selected like in clinical trials. This causes differences regarding group's compositions, significantly different in several characteristics, as Hospital del Mar is a reference center to elite athletes. These differences are also mentioned in other studies [14] [23]. Although the measurements were conducted by two experienced professionals with high interobserver agreement, human error may have occurred, particularly because of the very narrow angles.

Additionally, when the tibia is rotated during the X-ray and the projection is not exactly lateral, the error measurement of the slope may increase. In traumatic patients it is harder to obtain good quality images than in non-traumatic. Lat- 
eral radiographs were used for PTS measurements, and the field of view was limited to the proximal diaphysis distally. The longitudinal tibial axis, which is essential for PTS measurement, was drawn on the lateral knee radiographs. However, the use of an expanded lateral radiograph would depict a closer approximation of the LAT and PTS measurements. As there is no gold standard in tibial slope measurement, the results are variable, just as prior literature showed [14] [21] [25].

\section{Conclusion}

The results found in this study showed not enough significant difference to support MTPS, LTPS or PTS individually as a risk factor for ACL tear. However, when comparing traumatic LCA study group with traumatic control group, extreme PTS $\left(>12^{\circ}\right)$ was associated with LCA but no differences were found when comparing non-traumatic groups, which could mean that the importance of extreme angles in the LCA rupture may be restricted to traumatic mechanisms. In all measure methods, previous injury (previous ACL tear) was related to a higher risk of ACL tear; and increased age and a traumatic mechanism to a lower risk.

\section{Acknowledgements}

The authors have no acknowledgements to disclose, and they received no funding for the work described in this article.

\section{Conflicts of Interest}

The authors have no conflicts of interest to disclose in relation to this article.

\section{References}

[1] Zantop, T., Petersen, W., Sekiya, J.K., Musahl, V., Fu, F.H. (2006) Anterior Cruciate Ligament Anatomy and Function Relating to Anatomical Reconstruction. Knee Surgery, Sports Traumatology, Arthroscopy, 14, 982-992. https://doi.org/10.1007/s00167-006-0076-Z

[2] Reiman, P.R. and Jackson, D.W. (1987) Anatomy of the Anterior Cruciate Ligament. In: Jackson, D.W., Drez, D., Eds., the Anterior Cruciate Deficient Knee, CV Mosby \& Co, St. Louis, 17-26.

[3] Kennedy, J.C., Weinberg, H.W. and Wilson, A.S. (1974) The Anatomy and Function of the Anterior Cruciate Ligament as Determined by Clinical and Morphological Studies. Journal of Bone and Joint Surgery, 56A, 223-225. https://doi.org/10.2106/00004623-197456020-00001

[4] Amis, A.A. and Dawkins, G.P. (1991) Functional Anatomy of the Anterior Cruciate Ligament. Fibre Bundle Actions Related to Ligament Replacements and Injuries. The Journal of Bone and Joint Surgery, 73, 260-267. https://doi.org/10.1302/0301-620X.73B2.2005151

[5] Girgis, F.G., Marshall, J.L. and Monajem, A (1975) The Cruciate Ligaments of the Knee Joint. Anatomical, Functional and Experimental Analysis. Clinical Orthopaedics and Related Research, 106, 216-231. https://doi.org/10.1097/00003086-197501000-00033 
[6] Arnoczky, S.P. (1983) Anatomy of the Anterior Cruciate Ligament. Clinical Orthopaedics and Related Research, 172, 19-25. https://doi.org/10.1097/00003086-198301000-00006

[7] DeMorat, G., Weinhold, P., Blackburn, T., Chudik, S. and Garrett, W. (2004) Aggressive Quadriceps Loading Can Induce Noncontact Anterior Cruciate Ligament Injury. American Journal of Sports Medicine, 32, 477-483. https://doi.org/10.1177/0363546503258928

[8] Haapasalo, H., Parkkari, J., Kannus, P., Natri, A. and Jarvinen, M. (2007) Knee Injuries in Leisure-Time Physical Activities: A Prospective One-Year Follow-Up of a Finnish Population Cohort. International Journal of Sports Medicine, 28, 72-77. https://doi.org/10.1055/s-2006-924039

[9] Schmitz, R.J., Kulas, A.S., Perrin, D.H., Riemann, B.L. and Shultz, S.J. (2007) Sex Differences in Lower Extremity Biomechanics during Single Leg Landings. Clinical Biomechanics, 22, 681-688. https://doi.org/10.1016/j.clinbiomech.2007.03.001

[10] Majewski, M., Susanne, H. and Klaus, S. (2006) Epidemiology of Athletic Knee Injuries: A 10-Year Study. Knee, 13, 184-188. https://doi.org/10.1016/j.knee.2006.01.005

[11] Frank, B., Bell, D.R., Norcross, M.F., Blackburn, J.T., Goerger, B.M. and Padua, D.A. (2013) Trunk and Hip Biomechanics Influence Anterior Cruciate Loading Mechanisms in Physically Active Participants. American Journal of Sports Medicine, 41, 2676-2683. https://doi.org/10.1177/0363546513496625

[12] Shen, L., Jin, Z.G., Dong, Q.R. and Li, L.B. (2018) Anatomical Risk Factors of Anterior Cruciate Ligament Injury. Chinese Medical Journal, 131, 2960-2967. https://doi.org/10.4103/0366-6999.247207

[13] Alentorn-Geli, E., Pelfort, X., Mingo, F., et al. (2015) An Evaluation of the Association between Radiographic Intercondylar Notch Narrowing and Anterior Cruciate Ligament Injury in Men: the Notch Angle Is a Better Parameter Than Notch Width. Arthroscopy, 31, 2004-2013. https://doi.org/10.1016/j.arthro.2015.04.088

[14] Wordeman, S.C., Quatman, C.E., Kaeding, C.C. and Hewett, T.E. (2012) In Vivo Evidence for Tibial Plateau Slope as a Risk Factor for Anterior Cruciate Ligament Injury: A Systematic Review and Meta Analysis. American Journal of Sports Medicine, 40, 1673-1681. https://doi.org/10.1177/0363546512442307

[15] Wang, Y.L., Yang, T., Zeng, C., Wei, J., Xie, D.X., Yang, Y.H., Long, H.Z., Xu, B., Qian, Y.X., Jiang, S.D. and Lei, G.H. (2017) Association between Tibial Plateau Slopes and Anterior Cruciate Ligament Injury: A Meta-Analysis. Arthroscopy, 33, 1248-1259. https://doi.org/10.1016/j.arthro.2017.01.015

[16] Sun, Y.H., Chen, L.X., Jiao, Z.D., Wang, L., Zhang, R.M., Fang, J. and Li, J. (2015) Age-Related Changes of Posterior Tibial Slope and Its Roles in Anterior Cruciate Ligament Injury. International Surgery, 101, 70-77.

[17] Dean, R., DePhillipo, N., Chahla, J., et al. (2021) Posterior Tibial Slope Measurements Using the Anatomic Axis Are Significantly Increased Compared with Those That Use the Mechanical Axis. The Journal of Arthroplasty, 37, 243-249.

https://doi.org/10.1016/j.arthro.2020.09.006

[18] Waiwaiole, A., Gurbani, A., Motamedi, K., Seeger, L., Sim, M.S., Nwajuaku, P. and Hame, S.L. (2016) Relationship of ACL Injury and Posterior Tibial Slope with Patient Age, Sex, and Race. Orthopaedic Journal of Sports Medicine, 4. https://doi.org/10.1177/2325967116672852

[19] Gianotti, M., Marshall, S., Hume, P. and Bunt, L. (2009) Incidence of Anterior cruciate Ligament Injury and Other Knee Ligament Injuries: A National Popula- 
tion-Based Study. Journal of Science and Medicine in Sport, 12, 622-627. https://doi.org/10.1016/j.jsams.2008.07.005

[20] Bjordal, J., Arnoy, F., Hannestad, B. and Strand, T. (1997) Epidemiology of Anterior Cruciate Ligament Injuries in Soccer. American Journal of Sports Medicine, 25, 341-345. https://doi.org/10.1177/036354659702500312

[21] Hudek, R., Schmutz, S., Regenfelder, F., Fuchs, B. and Koch, P.P. (2009) Novel Measurement Technique of the Tibial Slope on Conventional MRI. Clinical Orthopaedics and Related Research, 467, 2066-2072. https://doi.org/10.1007/s11999-009-0711-3

[22] Hashemi, J., Chandrashekar, N., Gill, B., et al. (2008) The Geometry of the Tibial Plateau and Its Influence on the Biomechanics of the Tibiofemoral Joint. Journal of Bone and Joint Surgery, 90, 2724-2734. https://doi.org/10.2106/JBJS.G.01358

[23] Kızılgöz, V., Sivrioğlu, A.K., Ulusoy, G.R., Yıldız, K., Aydın, H. and Çetin, T. (2019) Posterior Tibial Slope Measurement on Lateral Knee Radiographs as a Risk Factor of Anterior Cruciate Ligament Injury: A Cross-Sectional Study. Radiograph, 25, 33-38. https://doi.org/10.1016/j.radi.2018.07.007

[24] Evans, J. and Nielson, J.L. (2021) Anterior Cruciate Ligament Knee Injuries. In: StatPearls, StatPearls Publishing, Treasure Island.

https://www.ncbi.nlm.nih.gov/books/NBK499848/

[25] Çullu, E., Aydogdu, S., Alparslan, B. and Sur, H. (2005) Tibial Slope Changes Following Dome-Type High Tibial Osteotomy. Knee Surgery, Sports Traumatology, Arthroscopy, 13, 38-43. https://doi.org/10.1007/s00167-004-0501-0

[26] Genin, P., Weill, G. and Julliard, R. (1993) The Tibial Slope. Proposal for a Measurement Method. Journal of Radiology, 74, 27-33.

[27] Fulton, J., Wright, K., Kelly, M., et al. (2014) Injury Risk Is Altered by Previous Injury: A Systematic Review of the Literature and Presentation of Causative Neuromuscular Factors. International Journal of Sports Physical Therapy, 9, 583-595.

[28] Jiang, C., Yip, K. and Liu, T. (1994) Posterior Slope Angle of the Medial Tibial Plateau. Journal of the Formosan Medical Association, 93, 509-512.

[29] Chiu, K.Y., Zhang, S.D. and Zhang, G.H. (2000) Posterior Slope of Tibial Plateau in Chinese. Arthroscopy, 15, 224-227. https://doi.org/10.1016/S0883-5403(00)90330-9

[30] Salmon, L., Russell, V., Musgrove, T., Pinczewski, L. and Refshauge, K. (2005) Incidence and Risk Factors for Graft Rupture and Contralateral Rupture after Anterior Cruciate Ligament Reconstruction. Arthroscopy, 21, 948-957 https://doi.org/10.1016/j.arthro.2005.04.110

[31] Lee, Y.S., Kim, J.G. and Lim, H.C. (2009) The Relationship between Tibial Slope and Meniscal Insertion. Knee Surgery, Sports Traumatology, Arthroscopy, 17, 1416-1420. https://doi.org/10.1007/s00167-009-0847-4 